\title{
L'instumentation dans le domaine X-UV
}

\author{
D. Schirmann
}

CEA/Centre d'Etudes de Limeil-Valenton, 94195 Villeneuve Saint Georges Cedex, France

During the past several years, rapid progress has been made in UV-soft X-ray instrumentation to study laser-created plasma emissions. To illustrate this advance, we describe present capabilities of ultra fast streak cameras and time gated microchannel plate imager tubes in ultra high speed photography, performance of photoconductive detectors for sensitive and high bandwidth measurements of various forms of radiation and capabilities of thinned CCD sensors illuminated from the back side to record UVsoft X-ray images.

\section{Introduction}

L'instrumentation dans le domaine X-UV (10 $\AA$ à $3000 \AA)$ s'est considérablement développée depuis dix ans, sous l'impulsion des besoins de l'astrophysique, des études des plasmas créés par laser et de leurs applications à la fusion par confinement inertiel, des études d'émission $\mathrm{X}$ stimulée, du développement des lasers à excimères...

Dans cet article, nous développons les progrès les plus récents et les plus remarquables réalisés en cinématographie ultrarapide dans le domaine des caméras à balayage de fente et des tubes obturateurs d'image, des photoconducteurs et de la détection d'images par dispositifs à transfert de charges (CCD amincis).

\section{La cinématographie ultra rapide}

\subsection{Les caméras à balayage de fente (CBF)}

Le principe de fonctionnement d'un tube de CBF est bien connu. Il consiste à transformer les photons incidents en électrons au moyen d'une photocathode, à extraire les photoélectrons créés au moyen d'une grille d'accélération et à les transporter vers un écran luminophore. Une optique électronique est utilisée pour former l'image électronique de la fente d'entrée de la CBF sur l'écran luminophore. Des plaques de déflexion permettent de défléchir les électrons en fonction du temps et de faire balayer l'écran luminophore par l'image électronique. L'image de l'écran est ensuite enregistrée sur un film photographique ou une caméra CCD via un bandeau de fibres optiques. L'utilisation de structures de tubes dites ouvertes par opposition aux tubes scellés permet de changer à la demande la nature de la photocathode et de détecter ainsi des photons dans un large domaine spectral allant des UV aux X-dur en adaptant l'épaisseur et la nature du matériau de la photocathode à l'énergie des photons détectés. La résolution temporelle d'un tube de CBF est donnée par l'expression suivante,

$$
\Delta \tau=\left(\Delta \tau_{1}^{2}+\Delta \tau_{2}^{2}\right)^{1 / 2}
$$

où $\Delta \tau_{1}$ et $\Delta \tau_{2}$ représentent respectivement la durée équivalente de la largeur de trace $\Delta \mathrm{x}$ à la vitesse de balayage la plus élevée vb sur l'axe temporel

$$
\Delta \tau_{1}=\Delta \mathrm{x} / \mathrm{vb}
$$

et la dispersion des temps de transit des photoélectrons $\Delta \tau_{2}$ liée à la nature de la photocathode et à l'énergie des photons détectés donc à la dispersion initiale des énergies des photoélectrons $\Delta \mathrm{E}$, et au champ électrique d'accélération $\mathrm{Eg}$ par la relation

$$
\Delta \tau_{2}=(2 \mathrm{~m} / \mathrm{e})^{1 / 2}(\Delta \mathrm{E})^{1 / 2} / \mathrm{Eg}
$$


L'amélioration des résolutions à la fois spatiale et temporelle des caméras porte sur la réduction de chacun de ces facteurs, notamment la réduction de la largeur de la trace, l'augmentation de la vitesse de balayage, la recherche de matériaux de photocathode à faible potentiel de sortie Vc, l'augmentation du champ électrique d'extraction (Tsakiris 1988) (Mens 1990) (Murnane 1991).

En France, l'effort soutenu depuis plus de deux décennies par la Direction des Applications Militaires avec le concours des sociétés Philips Composants, Thomson et A.R.P. a permis une progression remarquable des performances des CBF à la fois dans le domaine de la lumière infrarouge, visible, UV et dans le domaine des rayons $\mathrm{X}$. La caméra la plus récente et la plus performante, la C850 X réalisée avec le soutien de l'ANVAR pour l'étude de l'interaction à haut flux des lasers subpicosecondes avec la matière est une caméra d'un type nouveau (Mens 1991). Elle utilise un tube bilamellaire P850X de diamètre $50 \mathrm{~mm}$, représenté sur la figure 1 et dont la particularité par rapport à un tube classique est d'avoir 2 lentilles electrostatiques indépendantes LQ et $F_{2}$ permettant d'optimiser séparément sa résolution spatiale et sa résolution temporelle. La largeur à mi hauteur de la trace $\Delta \mathrm{x}$ mesurée en mode non balayé est seulement de $50 \mu \mathrm{m}$ pour une vitesse de balayage $\mathrm{v}_{\mathrm{p}}$ de $23 \mathrm{ps} / \mathrm{mm}$ de sorte que $\Delta \tau_{1}=1,38 \mathrm{ps}$. Le champ électrique d'extraction a été porté à $50 \mathrm{kV} / \mathrm{cm}$. Pour des photons de $1 \mathrm{keV}$ et une photocathode en iodure de césium CsI, le terme $\Delta \mathrm{E}$ est égal à $1,06 \mathrm{eV}$ ce qui donne $\Delta \tau_{2}=0,71 \mathrm{ps}$. La résolution temporelle de la caméra s'écrit donc

$$
\Delta \tau=\left(1,38^{2}+0,71^{2}\right)^{1 / 2}=1,6 \mathrm{ps}
$$

Cette caméra a été caractérisée avec une photocathode en palladium en utilisant un faisceau laser de 60 fs de durée d'impulsion et de $320 \mathrm{~nm}$ de longueur d'onde développé à I'ENSTA (Palaiseau) (Mens 1990) (Mens 1992). La figure 2 représente un double enregistrement de deux impulsions laser séparées de 4,4 ps. En réduisant progressivement cette intervalle de temps, nous avons pu vérifier que la résolution temporelle de cette caméra est inférieure à $2 \mathrm{ps}$ à cette longueur d'onde. La figure 2 montre également que l'image d'une grille de $15 \mathrm{pl} / \mathrm{mm}$ placée devant la photocathode est résolue avec un contraste supérieur à $40 \%$.

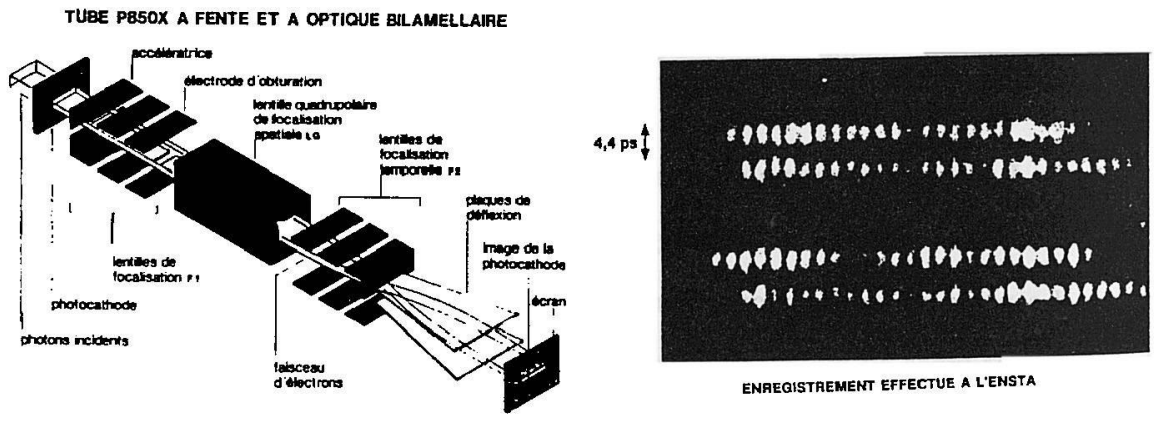

Fig. 1 Schéma du tube P850X

Fig. 2. Double enregistrement de deux impulsions de 60 fs de durée séparée de 4,4 ps à $310 \mathrm{~nm}$

Cet instrument, qui possède à la fois une résolution temporelle inférieure à 2 ps et une résolution spatiale supérieure à $15 \mathrm{pl} / \mathrm{mm}$, est l'un des plus performants existant sur le marché. Il est très bien adapté à l'étude des émissions X-UV des plasmas créés par interaction laser-matière avec des lasers subpicosecondes (Gauthier 1992) (Louis Jacquet 1992).

\section{2 Les tubes obturateurs à galette de microcanaux pulsées (GMC)}

Au cours des cinq dernières années, des progrès très importants ont été réalisés dans le domaine de l'imagerie à temps de pose très courts grâce à l'utilisation de GMC pulsées, notamment sous l'impulsion de J. Kilkenny (Kilkenny 1991) au LLNL (CA). Cette technologie permet d'atteindre actuellement des temps d'exposition inférieurs à $100 \mathrm{ps}$ avec une mise en oeuvre plus simple que celle des tubes électroniques à convertisseurs d'images. 
Un schéma de principe des tubes obturateurs à GMC pulsées est représenté sur la figure 3 : Un dépôt approprié d'un matériau métallique ou alcalin sur la face avant d'une GMC est utilisé comme photocathode pour convertir les photons X-UV en photo-électrons. Selon un modèle simple développé par Eberhardt 1979, une tension appliquée entre les faces avant et arrière de la GMC permet d'établir un gain en photoélectrons dans les microcanaux de la forme :

$$
\mathrm{G}=\mathrm{Go} . \mathrm{Vg}
$$

où Go est le gain initial éventuellement fixé par une tension de prépolarisation, $\mathrm{V}$ l'impulsion de haute tension appliquée et $\mathrm{g}$ est une constante voisine de 9 pour les GMC usuelles de rapport $\mathrm{L} / \mathrm{D}=40$, où $\mathrm{L}$ désigne l'épaisseur de la galette ici égale à $0,5 \mathrm{~mm}$ et $\mathrm{D}$ le diamètre de chaque microcanal égal à $12,5 \mu \mathrm{m}$. Pour une impulsion de haute tension de forme gaussienne de largeur à mi-hauteur $2 \mathrm{~T}_{1 / 2}$ de la forme

le gain s'écrit :

$$
V(t)=V_{0} \exp -\left(\ln 2\left(t / T_{1} / 2\right)^{2}\right)
$$

$$
\mathrm{G}(\mathrm{t})=\mathrm{Go} V_{\mathrm{o}} \mathrm{g} \exp -\left(\ln 2 \mathrm{~g} 1 / 2\left(\mathrm{t} / \mathrm{T}_{1 / 2}\right)^{2}\right)
$$

en supposant le temps de transit des photoélectrons dans les canaux négligeable devant la durée de l'impulsion $2 \mathrm{~T}_{1 / 2}$.

Ainsi, la largeur du gain à mi-hauteur est $2 \mathrm{~T}_{1 / 2} / \mathrm{g}^{1 / 2}$. Pour $\mathrm{g}=9$, la largeur du gain à mi-hauteur est seulement $1 / 3$ de la largeur à mi-hauteur de la tension appliquée, en raison de lalnon linéarité du gain par rapport à la haute tension. Ainsi, pour une impulsion gaussienne de haute tension appliquée de $300 \mathrm{ps}$ de durée à mi-hauteur, la durée du gain à mi-hauteur de la GMC sera seulement de 100 ps. Ce raisonnement montre comment la non-linéarité du gain avec la haute tension peut être utilisée pour relâcher les spécifications sur la durée de l'impulsion de haute tension difficile à réaliser dans cette gamme de temps.

Une tension continue $\mathrm{V}_{e}$ appliquée entre la face arrière de la GMC et un écran luminophore accélère les électrons secondaires vers l'écran, où ils sont convertis en photons visibles enregistrés ensuite avec un film ou une caméra $C C D$ via un pavé de fibres optiques. La résolution spatiale dans ce type de dispositif dit de focalisation à effet de proximité est maintenue en s'assurant que la vitesse transversale des électrons secondaires à la sortie des microcanaux reste négligeable devant la vitesse acquise dans l'espace GMC/écran de longueur L. Elle est donnée par l'expression :

$$
\delta=4 \mathrm{~L}(\Delta \mathrm{E} / \mathrm{eV})^{0,5}
$$

où, $\Delta \mathrm{E}$ désigne l'énergie initiale des photoélectrons (Henke 1981). Pour des valeurs typiques de $\mathrm{V}_{\mathrm{e}}=3 \mathrm{kV}, \mathrm{L}=0,5 \mathrm{~mm}$ et $\Delta \mathrm{E}=1 \mathrm{eV}, \delta=36 \mu \mathrm{m}$.

La haute tension pulsée est appliquée à la GMC grâce à des pistes conductrices déposées directement sur la face avant, jouant à la fois le rôle de photocathode et de lignes de transmission. Le choix du matériau déposé et des dimensions de la piste résultent de compromis entre d'une part l'efficacité de conversion de la photocathode et sa résistivité, qui dépendent de la nature du matériau et de l'épaisseur de la piste, et d'autre part l'impédance caractéristique de la ligne qui augmente quand la largeur de la piste diminue. Les tubes à GMC pulsées usuels ont des lignes en or d'impédance caractéristique 25 ou $12,5 \Omega$ et de largeur respective 6,4 ou $15 \mathrm{~mm}$ avec une épaisseur de $2000 \AA$ assurant une bonne efficacité quantique de détection (de l'ordre de quelques \% pour des photons de $1,5 \mathrm{keV}$ ).

Les applications des tubes à GMC pulsées dans les expériences de fusion par confinement inertiel, d'émission X stimulée ou de spectroscopie menées dans divers laboratoires sont nombreuses (Failor 1991), (Kilkenny 1991), (Azechi 1989). (LLE 1989). A titre d'exemple, la figure 4 représente une caméra $X$ muti-images réalisée au CEL-V (Schirmann 1992) à partir de 8 sténopés de $10 \mu \mathrm{m}$ de diamètre formant chacun l'image X d'un plasma sur l'une des 8 pistes de $25 \Omega$ déposées sur la face avant d'un tube obturateur d'images à GMC pulsée (SLIX 8) développé par Kentech Inc. (Grande Bretagne). La figure 5 représente (à droite) une succession d'images de l'implosion d'un microballon de $300 \mu \mathrm{m}$ de diamètre réalisée avec le laser Octal, espacées de 200 ps chacune par affichage de retards électriques entre l'arrivée successive des impulsions de haute tension sur chaque piste et dont le temps d'exposition était de 100 ps environ. La partie gauche représente une photographie de la même implosion prise avec une CBF de type Thomson TSN 506P munie d'un tube ouvert Philips P552X dont la fente était orientée suivant le diamètre du microballon. La complémentarité de ces deux enregistrements est évidente, l'un donnant continúument l'évolution spatio-temporelle d'une implosion suivant une dimension, l'autre une succession d'images à deux dimensions à des instants discrets. 


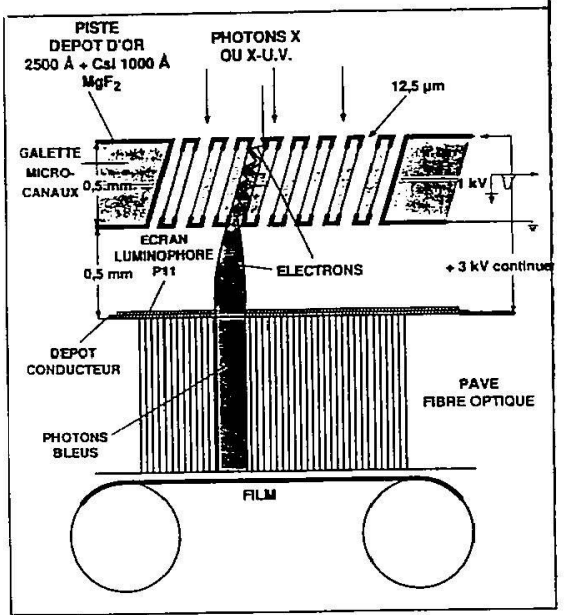

Fig. 3. Schéma d'un tube à GMC pulsée

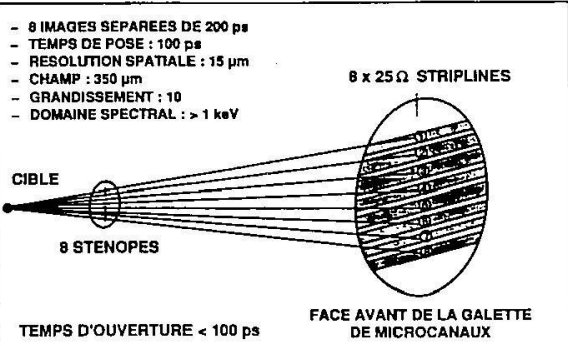

Fig. 4. Schéma d'une caméra multi-images

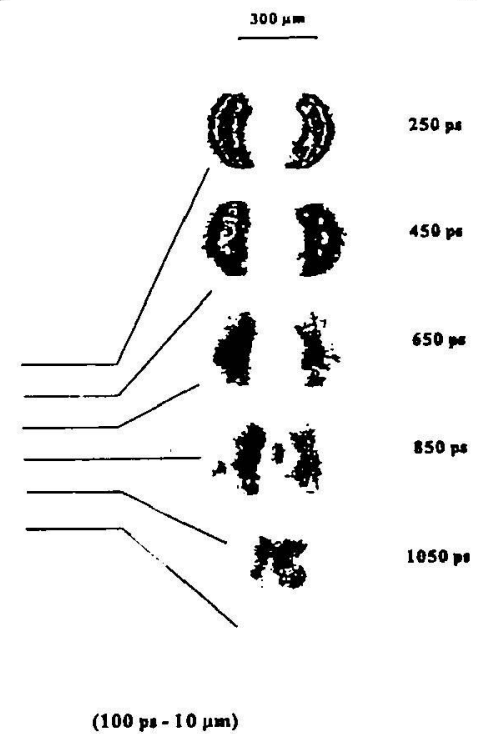

Fig. 5 Enregistrements de l'implosion d'un microballon de $300 \mu \mathrm{m}$ de diamétre avec une CBF (à gauche) et la caméra multi-images (à droite)

\section{Les photoconducteurs (PCD)}

Les matériaux PCD présentent des propriétés intéressantes pour détecter du rayonnement X-UV. Dans son principe, un détecteur à photoconducteur est constitué d'un petit morceau de matériau semiconducteur de quelques $\mathrm{mm}^{3}$ de volume (arséniure de gallium AsGa, diamant, tellure de cadmium CdTe...) polarisé par une source de tension extérieure. Le rayonnement incident est absorbé par effet photoelectrique en créant des paires électron- trou, lesquelles dérivent sous l'effet du champ électrique appliqué et font apparaître un courant (ou une augmentation) dans le circuit extérieur. L'absence de zone morte permet de détecter des photons de faible énergie, le seuil de détection étant fixé par l'énergie moyenne $\gamma$ nécessaire pour créer une paire électron-trou. L'évolution temporelle de leur densité n peut être modélisée par l'équation (Moulin 1989) (Pan 1990) (Kania 1990) (Kania 1991) (Ching 1991):

$$
\mathrm{dn} / \mathrm{dt}=\mathrm{g}(\mathrm{t})-\mathrm{n} / \tau
$$


où $g(t)$ représente le nombre de paires d'électron-trou créées par unité de volume égal au produit du nombre $F(t)$ de photons d'énergie Eph absorbés par unité de temps par le rapport $\mathrm{Eph} / \gamma$.

La sensibilité $S$ en $A / W$ peut être déduite de cette équation en supposant $\tau$ négligeable devant la durée de l'impulsion de photons

$$
S=(e / \gamma) \mu \tau V_{o} / L^{2}
$$

où $\mu$ est la mobilité des électrons, Vo la tension appliquée et $L$ la distance interélectrode. L'ordre de grandeur de $\mathrm{S}$ est de $6 \times 10^{-4} \mathrm{~A} / \mathrm{W}$ pour des valeurs de $\mathrm{t}$ de l'ordre de $100 \mathrm{ps}$.

La réponse à un photon absorbé donne naissance à une impulsion de courant $i(t)$ parcourant le circuit extérieur égale à :

$$
\mathrm{i}(\mathrm{t})=\left(\mathrm{e} \mu(\mathrm{Eph} / \gamma) \mathrm{Vo} / \mathrm{L}^{2}\right) \exp (-\mathrm{t} / \tau)
$$

Cette expression montre que la rapidité du détecteur est essentiellement liée à la durée de vie des porteurs, dont quelques valeurs sont données dans le tableau 1.

Tableau 1 : Valeurs de $\tau$ pour quelques PCD utilisés pour leur rapidité.

\begin{tabular}{|c||c|}
\hline \multicolumn{1}{|c||}{ matériau } & durée de vie $\tau$ (ps) \\
\hline \hline AsGa préirradié aux neutrons & 50 \\
\hline AsGa dopé Cr & 100 \\
\hline CdTe polycristallin & 10 \\
\hline InP dopé Fe & 200 \\
\hline diamant monocristallin & 200 \\
\hline
\end{tabular}

A titre d'exemple, la figure 6 représente un détecteur en CdTe réalisé par le LETICENG (France) pour détecter des rayons X. Il est constitué d'une couronne de diamètre intérieur $1 \mathrm{~mm}$ et extérieur $3 \mathrm{~mm}$, de $1 \mu \mathrm{m}$ d'épaisseur en CdTe polycristallin déposé par CVD (chemical vapor deposition) sur un substrat d'alumine d'épaisseur de $130 \mu \mathrm{m}$, monté en bout d'un câble coaxial de large bande passante $(30 \mathrm{GHz})$ de $3,6 \mathrm{~mm}$ de diamètre extérieur (type KS2). Les contacts du CdTe avec l'âme et l'extérieur du câble coaxial sont assurés par deux dépôts d'or concentiques. L'épaisseur de $1 \mu \mathrm{m}$ de CdTe a été choisie pour limiter la bande spectrale de détection à des photons $\mathrm{X}$ d'énergie inférieure à $3 \mathrm{keV}$, comme le montre la figure 7 qui donne la courbe d'efficacité d'absorption des photons en fonction de leur énergie. La coupure vers les basses énergie est fixée par la fonction de transmission d'un filtre de $5600 \AA$ d'aluminium déposé sur $4000 \AA$ de polystyrène $(\mathrm{CH})$. Le système d'enregistrement utilisé est représenté sur la figure 8. La tension de polarisation est appliquée aux bornes du photoconducteur au moyen d'un Té de polarisation de large bande passante. L'enregistrement du signal est effectué au moyen d'un oscilloscope IN 7000 de $7 \mathrm{GHz}$ de bande passante. Ce type de détecteur est bien adapté pour étudier l'émission $X$ des plasmas créés par laser.

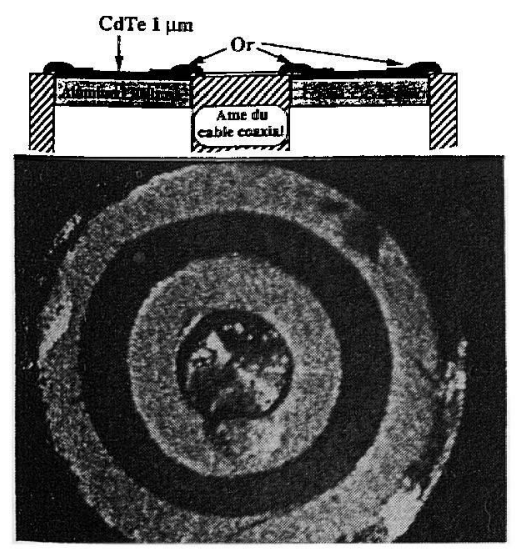

Fig. 6. Photographie du PCD

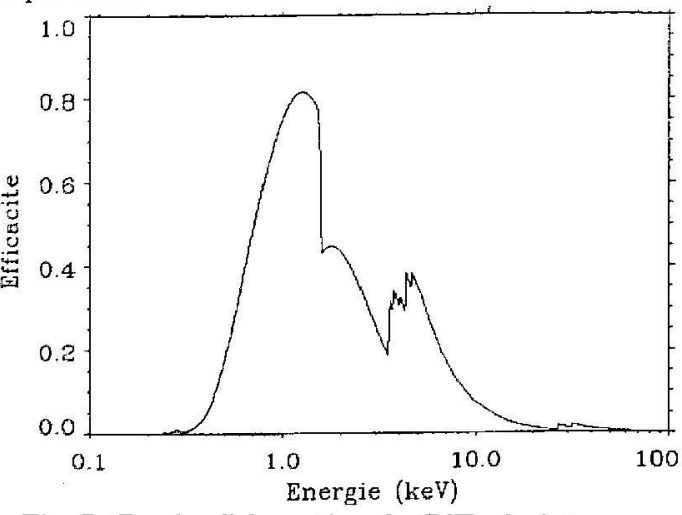

Fig. 7. Courbe d'absorption du CdTe de $1 \mu \mathrm{m}$ d'épaisseur placé derrière un filtre de $5600 \AA$ d'Al déposé sur $4000 \AA$ de polystyrène 
La figure 9 montre un enregistrement de l'émission $X$ d'un plasma créé en focalisant l'un des deux faiceaux du laser Phébus sur une cible en or pour une durée d'impulsion 200 ps à $10^{14} \mathrm{~W} / \mathrm{cm}^{2}$. On constate que la forme de l'impulsion $\mathrm{X}$ suit bien la forme de l'impulsion laser.

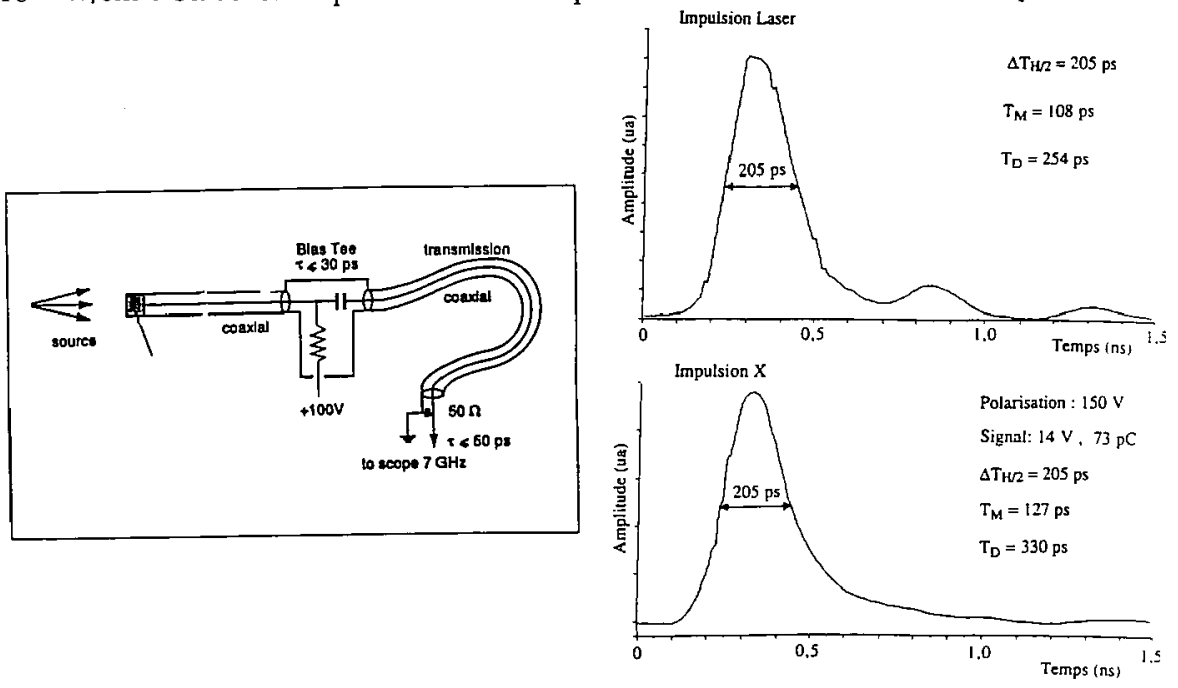

Fig. 8. Schéma du système d'enregistrement Fig. 9. Forme temporelle de l'émission $\mathrm{X}$ d'un plasma créé par laser enregistré avec un $\mathrm{PCD}$ en CdTe

Une évaluation de la sensibilité faite à partir d'un spectre enregistré avec le spectromètre $\mathrm{X}$ multi-voies à large bande Demix installé sur la chambre d'expériences Phébus donne pour valeur moyenne $2,2.10^{-22} \mathrm{C} /(\mathrm{keV} . \mathrm{V})$ entre 0,5 et $3 \mathrm{keV}$, soit 6,6 fois plus sensible qu'une photodiode à photocathode en or utilisée dans la même bande spectrale. La réponse de ce type de détecteur est linéaire sur plusieurs décades, comme l'a montré Kania 1990 avec un détecteur en diamant .

Les PCD devraient trouver de nombreuses applications dans les mesures temporelles de l'émission $X$ des plasmas en raison de leur rapidité ( $<100$ ps pour certains), de leur grande sensibilité et de leur grande dynamique.

\section{L'acquisition d'images X-UV par caméras à $\mathrm{CCD}$ amincis}

L'imagerie par CCD est couramment utilisée depuis de nombreuses années pour la détection d'images dans le domaine infrarouge, visible et, plus récemment dans le domaine des rayons X-dur (>1 keV) (Cavailler 1984) (Koppel 1977) (Pina 1991). La détection du rayonnement $U V$ lointain et des rayons $\mathrm{X}$-mou $(<1 \mathrm{keV})$ restait impossible avec les CCD usuels en raison de la présence, sur la face avant, d'une couche de verre protectrice $\left(\mathrm{SiO}_{2}\right)$ de quelques milliers Å d'épaisseur et des électrodes de collection des charges, empêchant la pénétration du rayonnement dans la zone sensible. La réalisation récente de CCD amincis, éclairés par la face arrière vient d'étendre leur domaine d'utilisation à pratiquement tout le spectre de la lumière $\mathrm{X}$ UV (Janesick 1984). Les CCD amincis sont des CCD dont on a éliminé le substrat situé en face arrière et fait subir un traitement de surface pour imposer un profil de potentiel entrainant la dérive vers la face avant (derrière laquelle se trouve la zone de collection des charges) des électrons créés en face arrière comme le montre la figure 10 (Tassin 1989). La figure 11 donne l'efficacité quantique de détection calculée en fonction de l'énergie des photons avec comme hypothèses une couche résiduelle de $100 \AA$ d'épaisseur de silice sur la face arrière et une épaisseur de $10 \mu \mathrm{m}$ de silicium pour la couche sensible (Salières 1992). Elle montre que cette structure est capable de détecter des photons X-UV dans une large gamme spectrale. 
THINNED CCD - Backside treatment
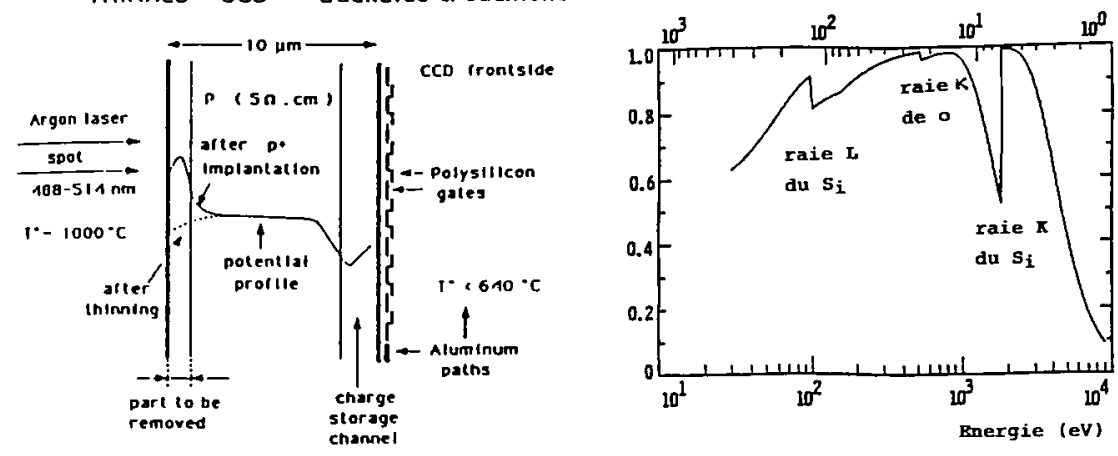

Fig. 10. Schéma d'un CCD aminci

Fig.11. Efficacité quantique d'interaction d'un $\mathrm{CCD}$ aminci (100 $\mathrm{A}$ de $\mathrm{SiO}_{2}$ et $10 \mathrm{~mm}$ de $\left.\mathrm{Si}\right)$

Nous avons caractérisé un CCD aminci comportant 512 x 512 pixels, type TH 7395 A, fabriqué par la société Thomson (TMS Saint Egrève France) associé à une caméra à sortie numérique sur 12 bits développée aux laboratoires Electroniques de Rennes (LER, ThomsonCSF), associée à une carte d'acquisition d'images spécifique commercialisée par la société A.R.P. (Strasbourg (France)). Pour cela, nous avons utilisé au laboratoire de l'Ecole Polytechnique (Palaiseau (France)) une installation de calibration pour rayons X-mou utilisant l'émission X d'un plasma créé par laser mise au point par Bénattar 1992. La figure 12 montre l'efficacité quantique totale de détection $Q E t$ en fonction de l'énergie des photons entre $60 \mathrm{eV}$ et $130 \mathrm{eV}$. QEt est reliée à QEi précédemment calculée par la relation

$$
\mathrm{QEt}=\mathrm{QEi} \times \mathrm{CCE} \times \mathrm{Eph} / 3,62
$$

où $\mathrm{CCE}$ désigne l'efficacité de collection des charges qui dépend étroitement de la valeur du champ électrique Es au voisinage de la face arrière et de leur vitesse de diffusion (ellemême fonction de la température d'utilisation du CCD) et du rapport Eph/3,62 de l'énergie du photon absorbé à l'énergie moyenne nécessaire pour créer une paire électron-trou dans le silicium. La discontinuité observée expérimentalement, vers $100,6 \mathrm{eV}$ correspond à la raie d'absorption $\mathrm{L}$ du silicium. Nous avons utilisé cette caméra $C C D$ pour enregistrer des spectres $\mathrm{X}$ entre 10 et $40 \AA$ émis par un plasma créé par laser derrière un spectromètre à transmission (Schirmann 1991). La comparaison (Figure 13) entre les spectres enregistrés avec un film sans gélatine, type Kodak SA 1, et un CCD TH 7395 A montre un très bon accord entre la réponse des deux détecteurs, validant ainsi l'utilisation du CCD aminci avec, de plus, un gain en sensibililé d'un facteur 500 en faveur du CCD.

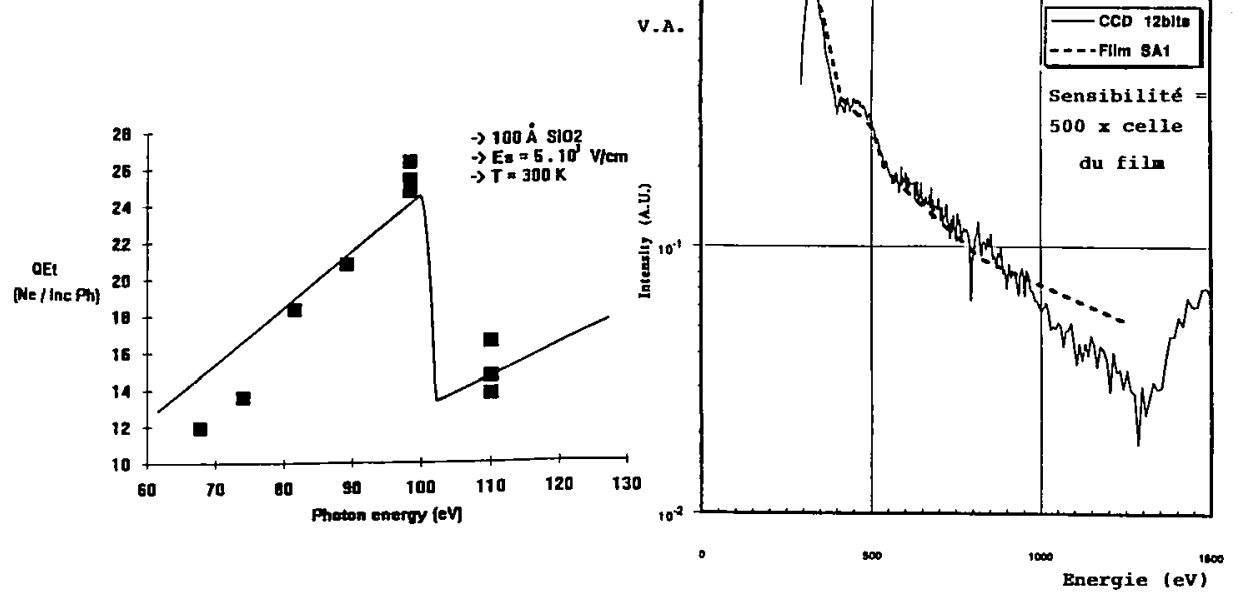

Fig; 12 Efficacité quantique totale mesurée ( ) et calculée (---)
Fig. 13. Comparaison d'un spectre enregistré avec un film SA1 et une camćra CCD aminci TH 7395 A 
Les CCD amincis devraient remplacer, dans de nombreux cas d'acquisition d'images, celle des films sans gélatine difficiles à se procurer, fort peu sensibles et délicats à manipuler.

\section{Conclusion}

L'instrumentation dans le domaine des X-UV a fait de nombreux progrès au cours de ces dernières années : dans le domaine de la cinématographie ultrarapide avec les caméras à balayage de fente dont les résolutions à la fois temporelle et spatiale peuvent atteindre 2 ps et 15 $\mathrm{pl} / \mathrm{mm}$ et les tubes obturateurs à galette de microcanaux pulsée qui permettent maintenant des

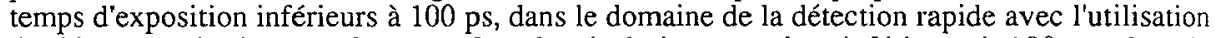
de détecteurs à photoconducteurs dont la résolution peut être inférieure à $100 \mathrm{ps}$, dans le domaine de l'acquisition des images avec la réalisation de CCD amincis 500 fois plus sensibles que les films sans gélatine usuels.

\section{Remerciements}

L'auteur remercie vivement J. L. Bocher, J. L. Bourgade, J. P. Le Breton, A. Mens, J. L. Miquel, M. Nail, P. Salières, R. Sauneuf du CEL-V pour leur participation aux nombreux résultats auxquels il a fait appel dans la rédaction de ce texte.

\section{Références}

[1]. Tsakiris, G.D. 1988 SPIE, High Speed Photography and Photonics, Vol. 1032, 910

[2]. Mens, A. et al. 1990 High Speed Photography and Photonics, Cambridge 90, SPIE vol. 1538

[3] Mens, A. 1992 8ème Conf. Intern. sur les phénomènes ultrarapides, Antibes (France)

[4]. Murnane, M. M. et al. 1990 Appl. Phys. Lett. 56, 20

[5]. Gauthier, J. C. et al. 1992 Femtosecond laser-induced plasma X-rays and Ionization Dynamics of High- $Z$ Materials

[6]. Louis Jacquet, M. et al , 1992 Japan-US Seminar, Kyoto (Japan)

[7]. Kilkenny, J. D. 1991 Laser and particle Beams, 9, 1, 49

[8]. Failor, B. H. et al. 1991 Rev. Sci. Instum. 62, 12, 2862

[9]. Henke, B. et al. 1981 J. Appl. Phys. 52, 1509

[10]. Eberhardt, E. H. 1979 Appl. Opt. , 1418 (1979)

[11]. Azechi, H. et al. 1989 Appl. Phys. Lett. 55, 10, 945

[12]. LLE 1989 Quartely Report DOE/DP40200-112, 41, 22 Rochester (NY)

[13]. Schirmann,D. 1992 Japan/US Seminar, Kyoto (Japan)

[14]. Kania,D. R. et al. 1990 Rev. Sci. Instrum. 61, 10, 2765

[15]. Pan, L. S. et al. 1990 Appl. Phys. Lett. 57, 6, 623

[16]. Moulin, H. 1989 Thèse Grenoble LETI/ CENG (France)

[17]. Kania, D. R. 1991 Laser and Particle Beams, 9, 1, 91

[18]. Ching, L. et al. 1991 E\&TR Sept-Oct , 15

[19]. Cavailler, C. et al. 1984 High Speed Photography Strasbourg SPIE, 491, 693

[20]. Koppel, L. N. 1977 Rev. Sci. Instr. 40, 669

[21]. Pina, L. et al. 1991 Laser and Particle Beams 6, 579

[22]. Janesick, J. et al. 1984 SPIE 501

[23]. Tassin, C. et al. 1989 Proc. ECO2 SPIE Paris (France),1140, 139

[24]. Schirmann, D. et al. 199121 th ECLIM Varssovie (Pologne) (à publier dans Laser and Particle Beams)

[25]. Salières, P. et al. 1992 3rd Int. Coll. on X-Ray Lasers Schliersee (Germany)

[26]. Benattar, R. et al. 1992 3rd Int. Coll. on X-Ray Lasers Schliersee (Germany) 